\title{
SUR LA RÉSISTANCE AU RAYONNEMMENT GAMMA DE L'ABEILLE OUVRIÈRE
}

\author{
PAR \\ G. COURTOIS et J. LECOMTE \\ Section des Applications des Radioéléments, Centre d'études \\ nucléaires, Saclay. \\ Station de Recherches Apicoles, Bures-sur-Ivette.
}

La très grande résistance des insectes aux radiations ionusantes est maintenant un phénomène bien connu. C'est ainsi que la dose léthale pour Ioo p. Ioo des sujets, lorsqu'elle est reçue en une seule fois, est de 600 Roentgens pour l'homme; de 800 pour la souris et de 80 ooo pour la Drosophile (PlOUgH, I952).

Cette résistance permet d'opérer des marquages à l'aide de radioéléments artificiels d'une assez forte activité sans craindre que les insectes présentent une réduction de leur durée de vie ou une modification importante de leurs comportements. Ces marquages, réalisés par de très nombreux auteurs permettent d'étudier de manière satisfaisante, les comportements des insectes.

Utilisant l'or $\operatorname{Ig} 8$ ( $(98$ Au) en suspension dans du sirop de sucre, nous avons procédé au marquage des individus composant une ruche d'Abeilles (Courtors et LECOMTE, I958). Nous avons employé dans ce but, une dose de 40 millicuries exposant ainsi chaque Abeille à environ 600 Roentgens. Cette dose n'a eu apparemment aucune influence sur le comportement immédiat de la ruche dansles deux essais effectués. Par contre, dans l'une de ces ruches, la reine a été remplacée par les ouvrières, dans le mois qui suivit l'application de la dose de radioactivité.

Bien qu'un plus grand nombre d'observations soit souhaitable, il est permis de se demander si ce remplacement n'est pas dû à une baisse de la fécondité de la reine. L'on sait que de pareils phénomènes se rencontrent quand survient une diminution de la ponte due à l'âge, une maladie ou toute autre cause.

Vottlant savoir dans quelles limites 1'Abeille pouvait supporter sans dommages apparents, l'effet du rayonnement, nous avons soumis 
des ouvrières à l'action de doses croissantes de rayonnement gamma. I.es irradiations ont été effectuées avec une source de 450 curies de cobalt radioactif $(60 \%)$, émetteur bêta de $0,306 \mathrm{MeV}$ et $2 \gamma$ par désintégrations de I,I7 et I,33 MeV). Le rayonnement $\beta$ étant autoabsorbé, seul le rayonnement $\gamma$ est à considérer.

I e dispositif d'irradiation est formé par un cylindre d'acier de So cm de diamètre rempli de plomb pour assurer une protection efficace et muni d'une cavité cylindrique verticale d'environ $15 \mathrm{~cm}$ de diamètre. Tout autour de cette cavité sont réparties d'une façon uniforme, cinq baguettes de cobalt, créant au centre de la cavité cylindrique une dose de rayonnement voisine de Iooo ooo $r$ /heure. Dans la cavité centrale se meut verticalement un cylindre qui permet d'introduire et de sortir les échantillons à irradier. sur ce cylindre, des protections de plomb placées en haut et en bas réduisent au minimum les dangers d'irradiations pour le manipulateur.

I.e volume utile est de l'ordre de $500 \mathrm{cc}$. I'homogénéité d'irradiation est assez bonne et varie de \pm Io $p$. Ioo autour de la valeur moyenne de 100 000 $r$ /heure. I es doses indiquées par la suite devront être comprises par conséquent comme susceptibles d'une telle variation en plus on en moins.

I,es Abeilles utilisées dans cet essai furent toutes des butineuses capturées à l'entrée de la ruche. I'on est ainsi assuré d'avoir des Abeilles d'âge honıgène puisque la fonction de butineuse n'est assumée que par les individus les plus âgés.

Ces Abeilles après avoir été capturées furent anesthésiées à l'aide d'anhydride carbonique et réparties par groupes de dix dans des cagettes. I.es cagettes de forme cylindrique avaient une base de 6 centimètres de diamètre et une hauteur de I,5 cm. A l'intérieur était contenu du candi à reine (mélange de miel et de sucre); de l'eau de boisson était également fournie. Vingt-quatre heures après leur introduction dans ces cagettes les Abeilles furent irradiées par la technique ci-dessus décrite. Selon les doses administrées, le temps de séjour dans l'irradiateur fut de II à I20 minutes et pour simplifier la présentation des résultats, $1 \mathrm{a} \mathrm{I}_{2} \mathrm{O}^{\mathrm{e}} \mathrm{mi}$ nute a été prise comme point de départ des temps de survie pour tous les échantillons. Durant toute l'irradiation, les Abeilles furent tenues à la température de $25^{\circ}$, ; par la suite, elles furent conservées en étuve à la température de $30^{\circ}$.

Deux cagettes témoins, peuplées dans les mêmes conditions ont été gardées enfermées pendant toute la durée de l'irradiation des cagettes d'essai dans un récipient métallique contenant la même quantité approximative d'air que l'irradiateur et placé à la même température.

Les résultats obtenus sont exprimés dans le tableau ci-joint. I'examen de ces résultats montre que l'on peut seulement être certain de 


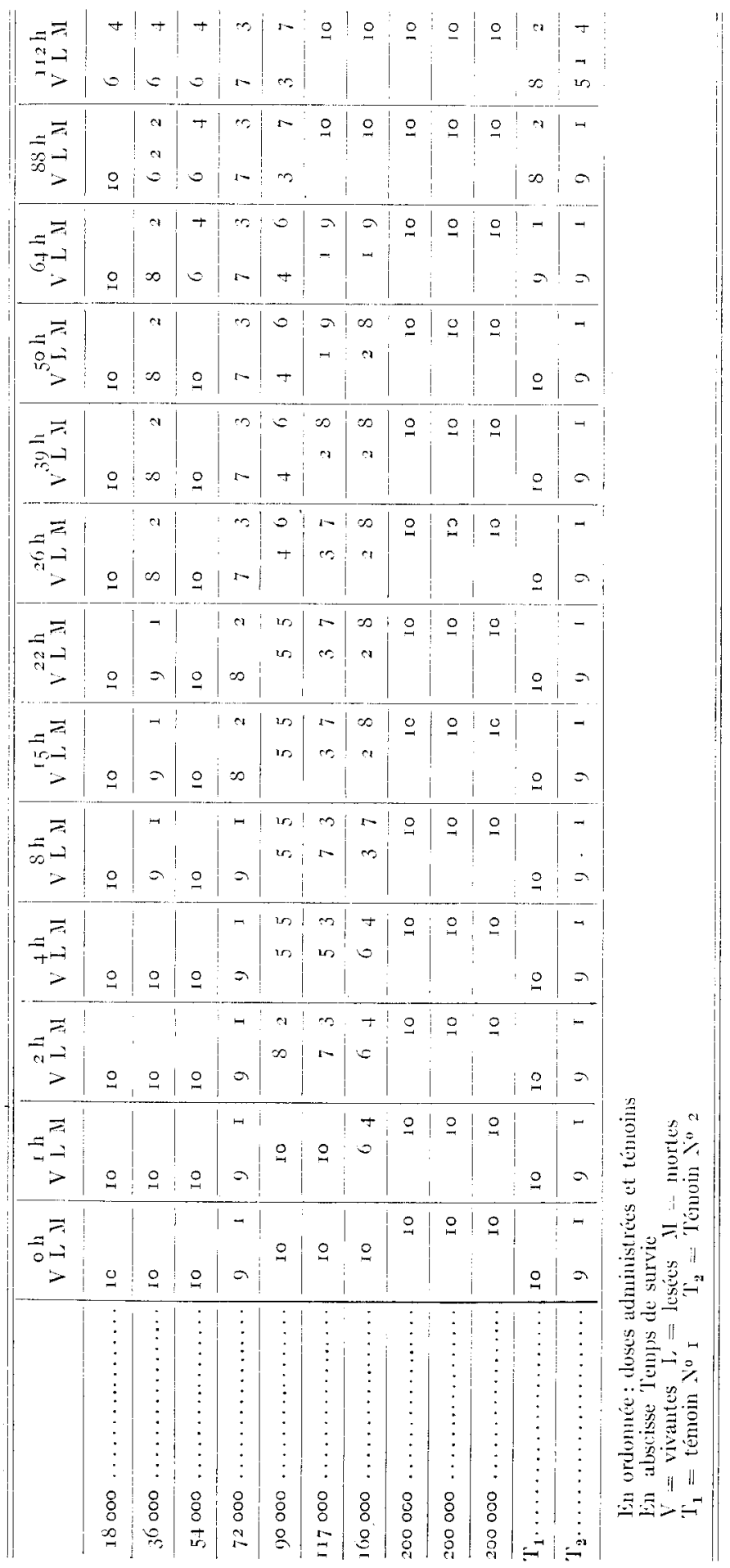


1'innocuité d'une dose égale à I 8 ooo Roentgens. I a dose de go ooo Roentgens se trouve la première à se manifester nocive d'une manière nettement significative. Enfin 200 ooo Roentgens tuent immédiatement tous les individus; dans notre essai, 30 ouvrières ont reçu cette dose.

L'étude de la survie n'a pas été poussée au-delà de ir 2 heures car à cette date, la mortalité des témoins est devenue relativement considérable. Cette mortalité n'a d'ailleurs rien d'anormal, si l'on considère que ces Abeilles ont passé r 26 hetres en cagettes depuis leur capture. Grassé et Chauvin (I944) ont obtenu des résultats analogues en étudiant la survie de butineuses placées dans des conditions comparables. Il est bon de signaler que les Abeilles survivantes du groupe ayant reçu une dose de 90 ooo $r$ après être passées par un stade d'immobjlité, ont retrouvé à la $39^{\mathrm{e}}$ heure la faculté de se déplacer normalement à l'intérieur de la cagette. Cependant, elles étaient incapables de voler tandis que les survivantes des groupes ayant reçu une dose de 72 ooo $r$ et au-dessous, se sont révélées jusqu'à la $88^{\circ}$ heure parfaitement capables de voler.

Une deuxième expérience fut mise en route pour déterminer la dose provoquant la première mortalité significative. En employant la même technique que précédemment, 48 Abeilles ouvrières furent soumises à une dose de 50 ooo Roentgens et 48 autres à 75 ooo Roentgens, 48 ouvrières étant réservées comme témoins.

Les résultats obtenus diffèrent notablement de ce que laissait espérer le premier essai et la mortalité fut très rapide avec les deux doses. Une heure après l'irradiation, il y avait II mortes dans le premier lot et 5 dans le second.

Au bout de 75 heures, les résultats étaient les suivants :

$50000 r=25$ vivantes et 25 mortes.

$75000 r=27$ vivantes et $2 \mathrm{I}$ mortes.

T'émoins $=47$ vivantes et $\mathrm{I}$ morte.

L es Abeilles ouvrières employées dans cette expérience ne provenaient pas de la ruche de celles utilisées dans le premir essai. Il s'agissait, de plus, d'abeilles maintenues dans des conditions bien particulières, dans une serre, sans communication avec l'extérieur. Ces Abeilles étaient certainement très âgées et dans de mauvaises conditions physiologiques et nous avons supposé que l'on pouvait inputer à ces raisons leur sensibilité relativement importante.

Une troisième expérience, destinée à vérifier le fait qu'il n'existait pas de grandes différences dans la sensibilité de colonies d'abeilles placées dans les mêmes conditions, fut effectuée avec des ouvrières provenant de deux ruches. Elles reçurent une dose uniforme de 50 ooo $r$. Dans ces conditions, la première abeille morte fut observée à 1a $26^{\mathrm{e}}$ heure après 1'irradiation. 
Au bout de 75 heures, les résultats suivants étaient obtenus :

Premieve ruche:

Irradiées vivantes : 57 Iésées : 2 Mortes: I

Témoins - : II - : $2-: 0$

Deuxiène ruche:

Irradiées Vivantes : 5 t Lésées : I Mortes : 5

Témoins - : $12-: 0 \quad-\quad: 0$

$\therefore i$ les résultats des essais 2 et 3 ne donnent pas beancoup plus de précisions sur la dose provoquant la première mortalité significative, ils ont par contre l'intérêt de nous montrer à quel point l'état physiologique peut rentrer en ligne de compte. I.es Abeilles de serre, pour des raisons inconnues présentent wne très grande sensibilité au raỹonnement par rapport aux Abeilles des trois autres ruches testées. Il est particulièrement intéressant de comparer ces résultats avec ceux obtenus par S'ULIIvax et Grosch (1953). Ces auteurs ont travaillé sur un hyménoptère du genre Habrobracon. I,irradiation avec un rayonnement X a donné les résultats suivants : I les doses égales ou inférienres à Iof́ i jo r ne produisent aucun effet visible. I,es doses de I $44200 r$ et de I $80250 r$ produisent nne diminution très sensible de tous les mouvements du corps, suivie assez rapidement d'un retour à la normale.

Par ailleurs, il est très curieux de constater que les radiations aux closes utilisées produisent un accroissement sensible de la durée de vie des individus par comparaison avec des témoins. İefficacité biologique relative des rayonnements gamma et $\mathrm{X}$ étant strictement comparable, l'on doit admettre que 1'Abeille possède une bien plus grande radiosensibilité que l'Habrobracon étudié.

Cette radiosensibilité n'empêche nullenent l'emploi des radioéléments aux fins de marquage, les doses reçues durant ces opérations étant très en dessonts des i 8 ooo $r$ considérés connne admissibles. Par contre, l'on ne peut envisager de se servir des radiations ionisantes conme moyen thérapeutique des maladies des Abeilles. STrDr.ER (H.) et STTCDER (R.) (1958) ont montré que des rayons contenant des larres mortes d'une atteinte de Ioque américaine étaient efficacement stérilisés par une dose de rayonnement ganma égale ou supéricure à roo ooo $r$. Cette dose serait compatible avec la résistance d'Habrobracon mais ne pourrait être supportée, nous l'avons vu, par l'Abeille.

\section{Résumón.}

I Abeile butineuse irradiée par le rayonnement gamma issu d'une source de Cobalt fo supporte sans dommages apparents nue dose de Is ooo $r$. Des donmages très apjréciables sont observés pour go ooo $r$. 
Une dose de $200000 r$ entraine la mort immédiate de Ioo p. Ioo des indi vidus. I'état physiologique de l'Abeille joue un rôle important dans 1a résistance au rayonnement gamma.

\section{REFERENCES BIBLIOGRAPHIQUES}

Covrtors (G.) et Lecoute (J.). - Sur un procédé de marquage des Abeilles butineuses au moven d'un radioisotope. $C . R$. Acad. Sc., 24\%, p. I47I 49, I958.

Grassí (P. P.) et Chauvix (R.). - L'effet de groupe et la survie des neutres dans les sociétés d'insectes. La revue scientifique, 82, 7, p. 46I-64, I944.

Plough (H. H.). - Radiation tolerances. Nucleonics, p. I6-20, august I952. Suldivax (R. L.) et Grosch (I). S.). - The radiation tolerance of an adult wasp. Nucleonics, 11, 3, p. 2I-23, march 1953.

StUdier (H.) et STUDI.ER (R.). - - The sterilization of American foulbrood with gamma rays. Minn. Beekpr., 11, I, p. 4-6, I058. 